\title{
Extremely sensitive chemical sensor for terahertz regime based on a hollow-core photonic crystal fibre
}

\author{
${ }^{1}$ Md. Selim Reza and ${ }^{2}$ Md. Ahasan Habib \\ ${ }^{1}$ Department of Electrical and Electronic Engineering, Bangabandhu Sheikh \\ Mujibur Rahman Science and Technology University, Gopalganj 8100, Bangladesh \\ selimreza.bsmrstu.eee@gmail.com \\ ${ }^{2}$ Department of Electrical and Electronic Engineering, Rajshahi University of \\ Engineering and Technology, Rajshahi 6204, Bangladesh \\ habib.eee.116.ah@gmail.com
}

Received: 09.01.2020

\begin{abstract}
We suggest a new kind of sensor based on a hollow-core photonic crystal fibre. It is aimed at identification of different chemicals using terahertz-range electromagnetic signals. Software based on a full-vectorial finite-element method is used to design this fibre and explore its propagation characteristics. By filling the core air hole with aqueous analytes and tuning different designing parameters at $2.4 \mathrm{THz}$, one can achieve the maximal relative sensitivities 98.5, 98.2 and 97.6\% respectively for benzene, ethanol and water analytes. Moreover, the confinement loss and the bulk material loss as low as $1.64 \times 10^{-13} \mathrm{~cm}^{-1}$ and $0.004 \mathrm{~cm}^{-1}$ can simultaneously be obtained under optimal conditions. The fibre suggested by us can be easily manufactured using modern fabrication techniques. We hope that our sensor can be efficiently used in many real-life applications.
\end{abstract}

Keywords: photonic crystal fibres, sensors, terahertz range, sensitivity, confinement loss

UDC: $535.8+621.391 .64$

\section{Introduction}

In the recent years, terahertz electromagnetic radiation range has achieved considerable attention due to its promising applications that include biomedical imaging, sensing, fast communications, cancer cell detection, astronomy etc. [1-3]. Note that we consider an electromagnetic signal as belonging to the terahertz range when its frequency changes from 0.1 to $10 \mathrm{THz}$ (see Ref. [4]).

One of important sensor applications is concerned with chemical sensing. Numerous types of chemicals are used as raw material in various industries. Some of them are mixed at fixed proportions to form a required raw solution for final products. If any mismatch occurs in that mixture, then the quality of the product will be deteriorated. Although there are many conventional techniques for identifying chemicals, they are often complicated and time-consuming. As an alternative, optical fibre-based sensors can be used in the detection process. In particular, over the last decades the researchers have proposed a number of sensors based upon photonic crystal fibres (PCFs) for identification of various gases and liquids. Different types of porous-core microstructure fibres have been devised [5-8], with the relative sensitivity typically not exceeding $75 \%$. Very recently, Islam et al. suggested a slotted-core kagome-lattice microstructure fibres [9] which offer the maximal relative sensitivity as high as $86 \%$ for the case of benzene. Although these fibres offer excellent guiding characteristics, the kagome lattice and the air hole of slotted type are very difficult to fabricate. Hollow-core PCFs (HC-PCFs) for chemical identification have 
also been reported in 2018 . The maximal sensitivity of the corresponding sensors is $96.8 \%$, which now represents the highest sensitivity reached for any chemical sensor that makes use of terahertz signals [10]. Very recently, an HC-PCF has been developed for chemical-spectroscopic applications [11]. It offers the relative sensitivity $89 \%$ under optimum conditions. A rectangulartype air hole is used in its design to create asymmetry in the core, and a small optical birefringence is inherent to its structure.

In the present work, we present a new hollow-core microstructure fibre for various chemicalspectroscopic applications, which employs terahertz signals. Here a hexagonal-type air hole is introduced into the core, which provides a larger area for photochemical interactions if compared with that reported in Ref. [11] for the rectangular-type hole. Moreover, trapezoidal-type air hole is introduced in the cladding, which reduces the actual bulk of material in our sensor. As a consequence, our HC-PCF appears to be lighter than those used in the other analogues of sensors. As it becomes clear from our further numerical analysis, the sensor described by us has high enough relative sensitivity $(98.5 \%)$ and very low confinement loss $\left(1.64 \times 10^{-13} \mathrm{~cm}^{-1}\right)$ at $2.4 \mathrm{THz}$, whenever the design conditions are kept at optimum levels. Moreover, our sensor offers very low effective absorption loss, $0.004 \mathrm{~cm}^{-1}$, under the same conditions. Finally, the HC-PCFs can be produced with no subtleties, using modern fabrication techniques.

\section{Methodology of design}

A 2D cross-sectional view of our sensor based on the HC-PCF is shown in Fig. 1. The hollow-core fibre contains a hexagonal-shaped air hole with the maximal diagonal length $D$ in the core region, since this type of core ensures better light confinement and larger core area than the other types of air holes such as rectangular, circular or elliptical ones. The cladding region is made of six trapezoidal air holes. Let the arm lengths of the air holes be given by the $\ell_{1}, \ell_{2}$ and $\ell_{3}$ parameters, and the distance between the two consecutive air holes in the cladding region (called as a strut) be equal to $p$. For simplicity, we express all the design parameters in terms of the $p$ value. They are as follows: $D=28.3 p, \ell_{1}=67 p, \ell_{2}=53 p$, and $\ell_{3}=14 p$. We have selected the above values so as to provide the best guiding characteristics (see the discussion in the next sections).

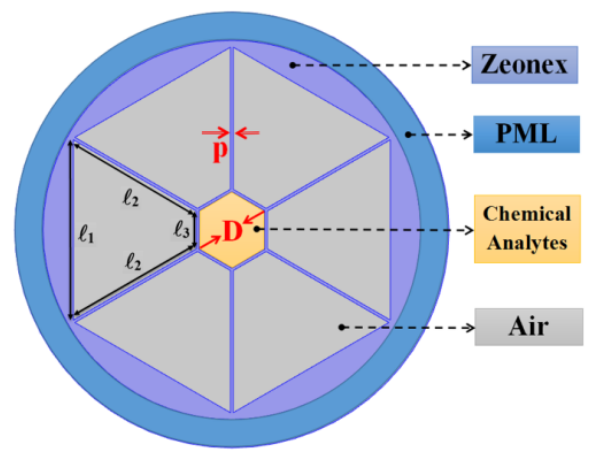

Fig. 1. Cross-sectional view of our HC-PCF-based sensor.

COMSOL Multiphysics v5.3a software associated with a standard full-vectorial finiteelement method has been used to visualize the work of our chemical sensor. A circular perfectly matched layer boundary condition is provided in the outer region of the cladding, which absorbs electromagnetic signal propagating towards the surface. ZEONEX (with the exact scientific name 'cyclic-olefin polymer') has been used as a basic material of our sensor, because it demonstrates excellent characteristics, if compared with many other materials. The bulk absorption loss of ZEONEX is $0.2 \mathrm{~cm}^{-1}$ in the region $0.1-10 \mathrm{THz}$, while its refractive index $(n=1.53)$ remains nearly

Ukr. J. Phys. Opt. 2020, Volume 21, Issue 1 
constant in the terahertz frequency range $[9,11]$. The refractive index of benzene, ethanol and water used as analytes in our study are respectively equal to $1.366,1.354$ and 1.33 (see Refs. [5-11]).

\section{Simulation results and discussions}

In case of all the types of PCFs, the light should be concentrated in the central region of optical waveguide. For better sensitivity of a sensor, a maximum light-analyte interaction is desirable inside the core. The tight confinement of the incident signal inside the core ensures the maximal interaction so that the sensitivity can become higher. The light-confinement characteristic of our fibre is illustrated in Fig. 2. It indicates that the dominating portion of light indeed travels inside the core.
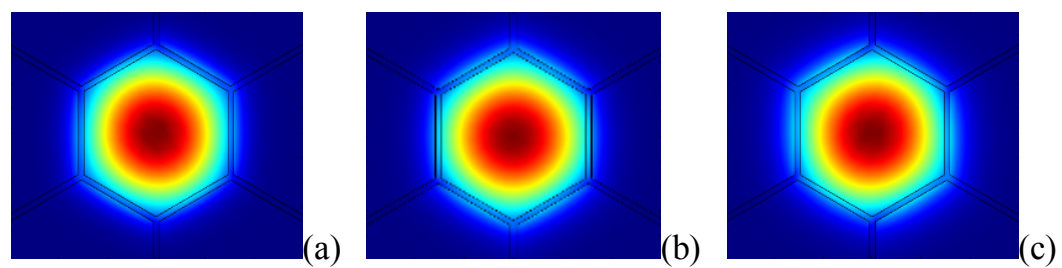

Fig. 2. Mode-field distributions in our HC-PCF-based sensor under optimal geometric conditions and at 2 THz, as calculated for the cases of (a) benzene, (b) ethanol and (c) water. The patterns obtained confirm that the light signal is tightly confined inside the central core region.

The most important parameter of any sensor is its relative sensitivity in detecting of a particular analyte or object. The relative sensitivity $r$ of an optical fibre-based sensor can be quantified as [5]

$$
r=\frac{n_{r}}{n_{e f f}} \times P,
$$

where $n_{\text {eff }}$ and $n_{r}$ denote the real parts of the effective refractive indices associated with the guided light and the sensing analyte, respectively. The $n_{\text {eff }}$ value represents the actual refractive index of the guide, for which the light signal propagates. It is adopted directly from the software. The term $P$ stands for the power fraction (in per cents) attributed to the fibre core only. Then the power fraction for any PCF can be expressed as [5]

$$
P=\frac{\int_{\text {sample }} R_{e}\left(E_{x} H_{y}-E_{y} H_{x}\right) d x d y}{\int_{\text {total }} R_{e}\left(E_{x} H_{y}-E_{y} H_{x}\right) d x d y} \times 100,
$$

where $E$ and $H$ are respectively the electric and magnetic fields, the subscripts indicate the directions of $x$ - and $y$-polarization modes, and $R_{e}$ indicates the real part of the complex quantity in either numerator or denominator. Here the numerator part yields the amount of power that interacts with the analyte sample, whereas the denominator corresponds to the same quantity taken for the total fibre.

The relative sensitivities of our HC-PCF calculated for three different analytes are shown in Fig. 3 as functions of electromagnetic frequency. The optimal core diagonal is taken to be $D=340 \mu \mathrm{m}$. The reasons behind this particular $D$ value will be discussed further on. All the plots in Fig. 3 show very similar characteristics for different analytes. The relative sensitivity increases with increasing operating frequency until the value of $2 \mathrm{THz}$ and then becomes almost unchanged when the frequency increases above this value. The characteristics of higher-frequency electromagnetic signals are responsible for this phenomenon, since they always try to propagate inside a zone of higher refractive index. As the refractive indices of air and analyte are respectively 
unity and a number greater than 1.33, still larger portion of light opts to go through the analyte with increasing frequency. As a result, a stronger light-analyte interaction takes place, which increases the relative sensitivity. The particular data of Fig. 3 demonstrates that the relative sensitivity of our HC-PCF-based sensor for benzene, ethanol and water is $98.5,98.2$ and $97.6 \%$ at $2.4 \mathrm{THz}$. This is better than the values reported in the previous works [5-11].
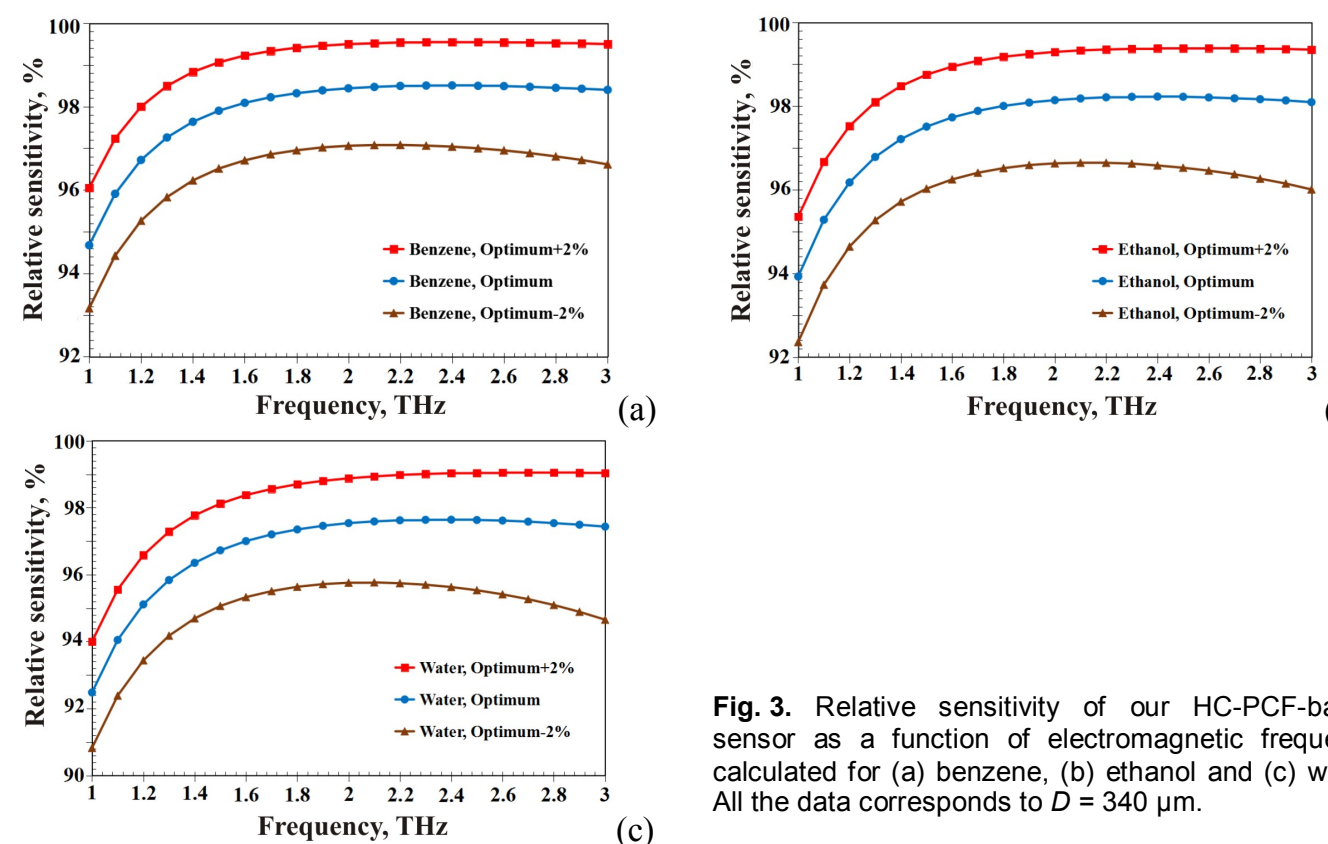

Let us discuss the other important guiding parameter called as a confinement loss. This loss indicates the amount of electromagnetic power captured by the cladding air holes that surround the core. For efficient operation, this loss of the sensor should be as low as possible. This parameter can be calculated via the relation [5]

$$
\alpha_{C L}=8.686 \times \frac{2 \pi f}{c} \operatorname{Im}\left(n_{e f f}\right),
$$

where $f$ represents the operating signal frequency, $c$ the light speed in free space, and $\operatorname{Im}\left(n_{\text {eff }}\right)$ the imaginary part of the effective refractive index for the guided mode. The confinement losses of our sensor calculated for different operating frequencies are displayed in Fig. 4. One can see that the loss decreases with increasing operating frequency (see the reasons mentioned in the previous section). The confinement loss of the sensor at $2.4 \mathrm{THz}$ is of the order of $10^{-13} \mathrm{~cm}^{-1}$ for all the three analytes (benzene, ethanol and water). This value is lower than those reported previously (see Refs. [5-11]).

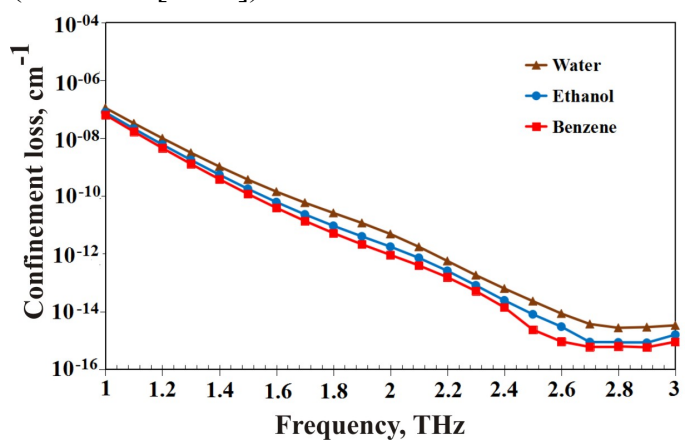

Fig. 4. Confinement loss of our fibre calculated at different operating frequencies and $D=340 \mu \mathrm{m}$.

Ukr. J. Phys. Opt. 2020, Volume 21, Issue 1 
Another kind of unavoidable loss, an effective material loss or a bulk material loss, occurs in all kinds of the optical sensors. This parameter indicates the amount of electromagnetic energy captured by the solid material present in the optical fibre. Notice that this loss is dependent on the loss coefficient of the background material. It is known that ZEONEX used in our sensor offers the lowest loss coefficient, which can be expressed as follows (see Ref. [11]):

$$
\alpha_{e f f}=\frac{\left(\varepsilon_{0} / \mu_{0}\right)^{1 / 2} \int_{A_{\operatorname{mat}}} n \alpha_{\text {mat }}|\mathrm{E}|^{2} d \mathrm{~A}}{2 \int_{A l l} S_{z} d \mathrm{~A}},
$$

Here $\alpha_{\text {mat }}$ represents the bulk material absorption loss, $\mu_{0}$ the permeability of free space, $\varepsilon_{0}$ the permittivity of air, $n$ the effective refractive index of the guided light, $E$ the modal electric field, $S_{z}$ the $z$-component of the Poynting vector, and $A$ the area of the fibre.

The effective material loss of our hollow-core fibre calculated at $D=340 \mu \mathrm{m}$ is shown in Fig. 5 as a function of operating frequency. It is clear from Fig. 5 that, at any particular frequency, the effective material loss is higher for the analytes with lower refractive indices. This happens because the refractive-index difference between the core and the cladding increases with increasing analyte's refractive index, so that a greater amount of light propagates in the core region and the loss decreases. It is known that the $\alpha_{\text {mat }}$ parameter is close to $0.2 \mathrm{~cm}^{-1}$ up to the frequency of $2 \mathrm{THz}$ and then it gets higher. That is why the effective material loss is also higher at the frequencies higher than $2 \mathrm{THz}$. As for specific figures, the effective material loss of our sensor at $2.4 \mathrm{THz}$ is equal to $4.46 \times 10^{-3}$, $4.98 \times 10^{-3}$ and $6.08 \times 10^{-3} \mathrm{~cm}^{-1}$ respectively for benzene, ethanol and water.

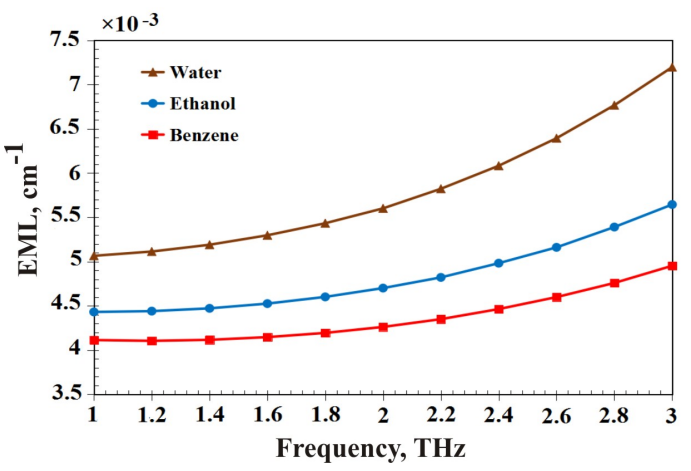

Fig. 5. Effective material loss of our fibre calculated at different operating frequencies and $D=340 \mu \mathrm{m}$.

The effective material loss and the confinement loss of the sensor are shown in Fig. 6 as functions of the core circumradius. It is evident that the two loss curves intersect each other when the core radius is equal to $170 \mu \mathrm{m}$. In other words, the optimum core diagonal at which the total loss becomes minimal amounts to $D=340 \mu \mathrm{m}$, as we have mentioned before.

Now we discuss in brief possible techniques for fabricating the HC-PCF described in the present study. Various techniques have been employed in the recent years for fabrication of porous-core and hollow-core fibres, including 3D printing, as well as stack and draw, sol-gel, drilling, capillary stacking, extrusion and some other techniques [9-11]. Among these methods, the extrusion technique can be used when implementing our fibre, because any kind of its structure can be fabricated with this technique. Moreover, a kagome-lattice fibre has been recently fabricated using the same technique. It has revealed a quite acceptable accuracy. These facts tell us that our fibre can also be fabricated in the same manner. It is the more so since the design of our HC-PCF is much easier than that of the kagome type (see Ref. [12]). Finally, the main guiding 
parameters typical for our fibre and some other remarkable structures reported in the literature are compared in Table 1. This comparison demonstrates that our fibre-based sensor looks better than the sensors reported previously.

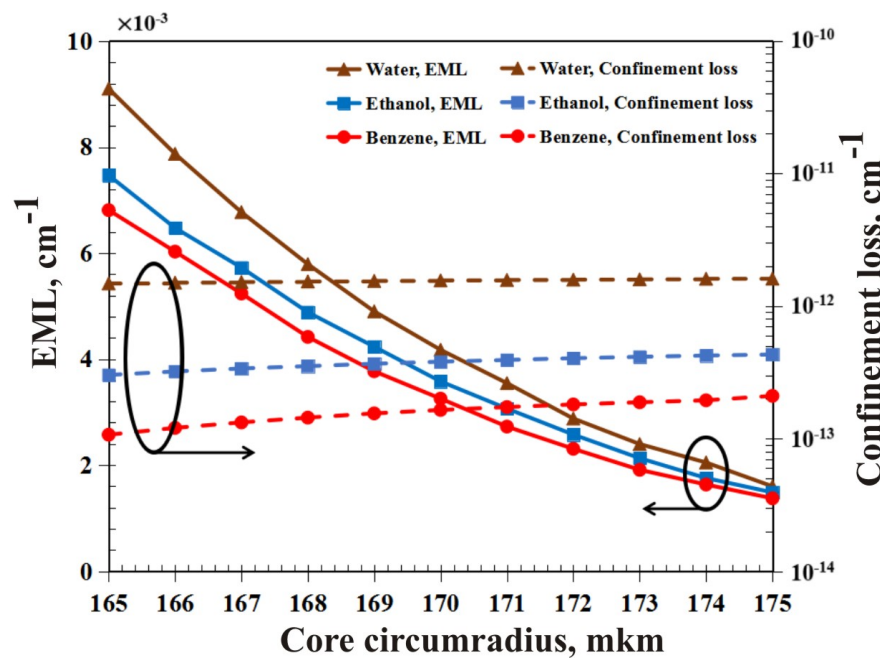

Fig. 6. Effective material loss and confinement loss of our HC-PCFbased sensor calculated for different core circumradii.

Table 1. Comparison of guiding characteristics typical for our HC-PCF-based sensor and some other known chemical sensors.

\begin{tabular}{c|cccc}
\hline Ref. \# & Year & $\begin{array}{c}\text { Relative } \\
\text { sensitivity, } \%\end{array}$ & $\begin{array}{c}\text { Effective material } \\
\text { loss } \alpha_{\text {eff }}, \mathrm{cm}^{-1}\end{array}$ & $\begin{array}{c}\text { Confinement loss } \\
\alpha_{C L}, \mathrm{~cm}^{-1}\end{array}$ \\
\hline$[5]$ & 2016 & 49.17 & - & $2.75 \times 10^{-10}$ \\
{$[6]$} & 2017 & 53.35 & - & $1.60 \times 10^{-10}$ \\
{$[7]$} & 2016 & 59.07 & - & $10^{-11}$ \\
{$[8]$} & 2017 & 65.18 & - & $5.90 \times 10^{-9}$ \\
{$[9]$} & 2018 & 85.70 & - & $7.21 \times 10^{-8}$ \\
{$[10]$} & 2018 & 96.80 & 0.0035 & $8.21 \times 10^{-13}$ \\
{$[11]$} & 2019 & 89.00 & - & $1.15 \times 10^{-7}$ \\
This & - & 98.50 & 0.0044 & $1.64 \times 10^{-13}$ \\
work & & & & \\
\hline
\end{tabular}

\section{Conclusion}

In this study, a new kind of HC-PCF-based chemical sensor is presented for detection of chemical analytes using terahertz signals. In order to design our fibre, a single hexagonal-type air hole and six trapezoidal air holes are introduced respectively into the core and the cladding. To the best our knowledge, this type of structure has never been reported as either a terahertz waveguide or a chemical sensor used in the terahertz regime. The sensor suggested by us exhibits a very high relative sensitivity. Namely, our calculations at the electromagnetic frequency $2.4 \mathrm{THz}$ testify the figures 98.5, 98.2 and 97.6\% respectively for benzene, ethanol and water. As far as we know, these are the best sensitivity figures reported up to date. Moreover, a very low confinement loss, of the order of $10^{-13} \mathrm{~cm}^{-1}$, and an extremely low effective material loss, $0.004 \mathrm{~cm}^{-1}$, can be achieved at particular design parameters. These excellent characteristics of our HC-PCF can warrant its applications in the sensing sectors of different industries.

\section{References}

1. Ho I C, Guo X and Zhang X C, 2010. Design and performance of reflective terahertz air biased-coherent-detection for time domain spectroscopy. Opt. Express. 18: 2873-2883. 
2. Habib M A and Anower M S, 2019. Design and numerical analysis of highly birefringent single mode fiber in terahertz regime. Opt. Fiber Technol. 47: 197-203.

3. Fischer B M, Hoffmann M, Helm H, Wilk R, Rutz F, Ostmann T K, Koch M and Jepsen P U, 2005. Terahertz time-domain spectroscopy and imaging of artificial RNA. Opt. Express. 13: 5205-5215.

4. Habib M A and Anower M S, 2019. Square porous core microstructure fiber for low loss terahertz applications. Opt. Spectrosc. 126: 607-613.

5. Asaduzzaman S, Ahmed K, Bhuiyan T and Farah T, 2016. Hybrid photonic crystal fiber in chemical sensing. Springer Plus. 5: 1-11.

6. Arif M F H and Biddut M J H, 2017. A new structure of photonic crystal fiber with high sensitivity, high nonlinearity, high birefringence and low confinement loss for liquid analyte sensing applications. Sens. Biosens. Appl. 12: 8-14.

7. Arif M F H, Ahmed K, Asaduzzaman S and Azad M A K, 2016. Design and optimization of photonic crystal fiber for liquid sensing applications. Photon. Sens. 6: 279-288.

8. Paul B K, Ahmed K, Asaduzzaman S and Islam M S, 2017. Folded cladding porous shaped photonic crystal fiber with high sensitivity in optical sensing applications: design and analysis. Sens. Biosens. Appl. 12: 36-42.

9. Saiful Islam Md, Sultana J, Ahmed K, Rakibul Islam M, Dinovitser A, Wai-Him Ng B and Abbott D, 2018. A novel approach for spectroscopic chemical identification using photonic crystal fiber in the terahertz regime. IEEE Sens. J. 18: 575-582.

10. Saiful Islam Md, Sultana J, Rifat A A, Dinovitser A, Wai-Him Ng B and Abbott D, 2018. Terahertz sensing in a hollow core photonic crystal fiber. IEEE Sens. J. 18: 4073-4080.

11. Habib M A, Anower M S, Abdulrazak L F and Reza M S, 2019. Hollow core photonic crystal fiber for chemical identification in terahertz regime. Opt. Fiber Technol. 52: 101933.

12. Falkenstein P, Merritt C D, and Justus B L, 2004. Fused performs for the fabrication of photonic crystal fibers. Opt. Lett. 29: 1858-1860.

Md. Selim Reza and Md. Ahasan Habib. 2020. Extremely sensitive chemical sensor for terahertz regime based on a hollow-core photonic crystal fibre. Ukr. J. Phys. Opt. 21: 8-14. doi: $10.3116 / 16091833 / 21 / 1 / 8 / 2020$

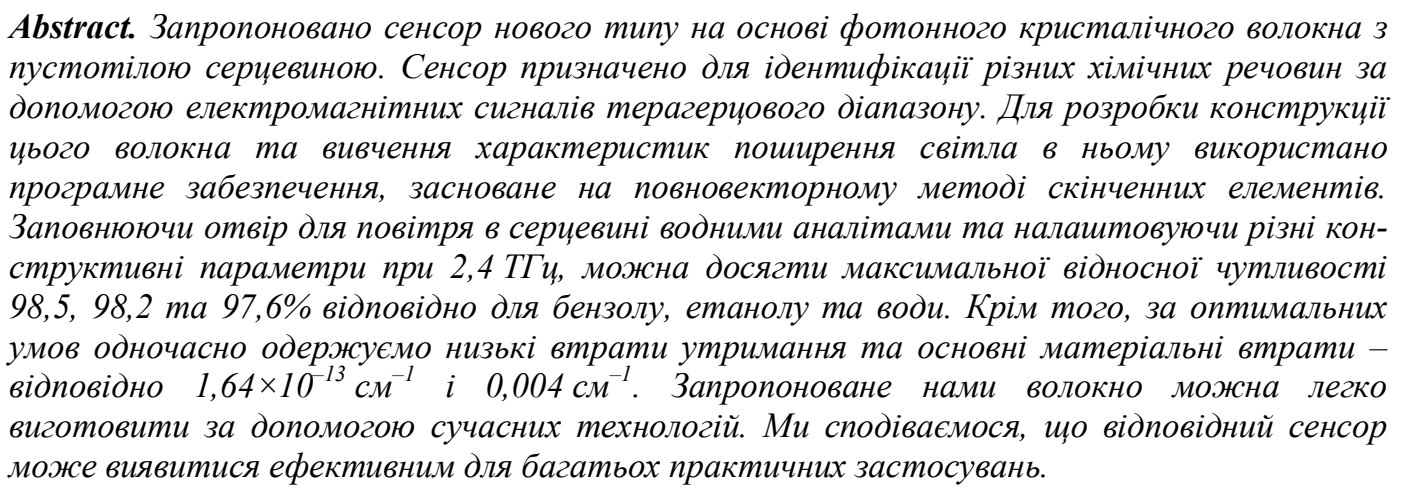

